Rev. Int. Contam. Ambie. 36 (4) 835-845, 2020

https://doi.org/10.20937/RICA.53600

\title{
EFECTOS DE LAS AGUAS RESIDUALES DE POZOS PETROLEROS SOBRE LOS SUELOS DE SABANAS UBICADAS CERCA DE EL FURRIAL, ESTADO MONAGAS, VENEZUELA
}

Effects of sewage fluids from oil drillings on the soils of savannas located near El Furrial, Monagas State, Venezuela

\author{
Danilo LÓPEZ-HERNÁNDEZ ${ }^{1 *}$, Carmen HERNÁNDEZ ${ }^{2}$, Fernando LIENDO ${ }^{3}$, \\ Juan URICH $^{4}$ y Oswaldo VALLEJO-TORRES ${ }^{5}$
}

${ }^{1}$ Centro de Ecología Aplicada, Instituto de Zoología y Ecología Tropical, Facultad de Ciencias, Universidad Central de Venezuela, Apartado Postal 47058, Caracas 1041-A, Venezuela.

${ }^{2}$ Universidad Nacional Experimental Marítima de Caribe, Ingeniería Ambiental, Apartado Postal 1162, Catia la Mar, Estado Vargas, Venezuela.

${ }^{3}$ Instituto de Tecnología Venezolana para el Petróleo, Apartado Postal 76343, Caracas 1070-A, Venezuela.

${ }^{4}$ Postgrado de Ecología, Facultad de Ciencias, Universidad Central de Venezuela, Caracas, Apartado Postal 47058, Caracas 1041-A, Venezuela.

${ }^{5}$ Decanato de Postgrado Universidad Nacional Experimental de los Llanos Occidentales, Apartado Postal 3350, Guanare, Estado Portuguesa, Venezuela.

*Autor para correspondencia: danilo.lopez@ciens.ucv.ve

(Recibido: abril de 2019; aceptado: enero de 2020)

Palabras clave: contaminación, sodio, bario, aluminio, fluidos de perforación.

\section{RESUMEN}

En los ambientes terrestres afectados por la actividad petrolera los fluidos de perforación y las aguas residuales (AR) generados en estas operaciones representan un problema ambiental y estético de importancia. Las AR, ricas en sólidos en suspensión, sales solubles y con potenciales contaminantes, son transportadas a una fosa donde se tratan con un agente floculante y se corrige su $\mathrm{pH}$. Posteriormente, las aguas resultantes son regadas al ambiente. Es importante conocer los efectos de esas AR sobre los ecosistemas terrestres circunvecinos. El objetivo de este trabajo es analizar los niveles de sales solubles y metales pesados en los suelos de sabanas adyacentes a pozos petroleros ubicados en el oriente de Venezuela después del riego con AR. Cuando son regadas al ambiente, las AR tratadas tienen un efecto encalante sobre la acidez natural de esos suelos. En ninguno de los casos estudiados se encontraron niveles altos de metales pesados ( $\mathrm{Fe}, \mathrm{Mn}, \mathrm{Cu}$ y $\mathrm{Zn}$ ) y aluminio soluble en los suelos, ya que fueron corregidos por el incremento de $\mathrm{pH}$. En general, los suelos tratados con los efluentes presentaron mayor concentración de sodio; sin embargo, no existe riesgo ambiental potencial en las cantidades de sodio soluble liberados a los ultisoles arenosos, ya que el buen drenaje interno de éstos y la adecuada precipitación de la zona permiten el pronto desalojo de sales sin peligro de salinización. No obstante, se recomienda una estricta vigilancia, en particular de los niveles de $\mathrm{pH}$ de las aguas residuales antes de ser regadas a las sabanas circunvecinas.

Key words: contamination, sodium, barium, aluminum, drilling fluids. 


\begin{abstract}
In terrestrial environments affected by oil operations, oil drilling and sewage fluids (SF) represent an environmental and aesthetic problem of importance. Before watered to the environment, SF rich in suspended solids and with potential contaminants, are transported to a tank treated with a flocculant agent and corrected for $\mathrm{pH}$. The resulting waters are then disposed to the local savannas. It is important to know the effects that those treated SF may have on the surrounding ecosystems. This contribution aims to analyze the levels of soluble salts and heavy metals in the soils adjacent to oil wells located in the eastern part of the Venezuelan savannas after irrigation with treated SF. The treated SF watered to the environment have a liming effect on the natural acidity of soils. In general, no high levels of heavy metals ( $\mathrm{Fe}, \mathrm{Mn}, \mathrm{Cu}$ and $\mathrm{Zn}$ ) were found nor soluble aluminum in treated soils, which is due to the rise in $\mathrm{pH}$ after treatment. The soils treated with effluents generally had a higher sodium concentration. However, there is no potential environmental risk in the amounts of soluble sodium released to the sandy ultisols since the good internal drainage of these and the proper precipitation of the area allow the soon eviction of salts without danger of salinization. Nevertheless, strict monitoring is recommended, in particular of the $\mathrm{pH}$ levels of wastewater before being irrigated to the surrounding savannas.
\end{abstract}

\section{INTRODUCCIÓN}

La industria petrolera es considerada como una actividad fuertemente generadora de problemas ambientales. En efecto, en las diferentes etapas de exploración, explotación, producción y transporte del crudo se generan desechos, los cuales deben disponerse y tratarse de forma ambiental efectiva debido a su efecto negativo sobre los diferentes componentes de los ecosistemas; de lo contrario, se convierten en pasivos ambientales (Arellano 2008). Asimismo, la producción petrolera implica el manejo y potencial derrame de hidrocarburos, los cuales son importantes contaminantes ambientales (Konečný et al. 2003, Gay et al. 2010, López-Hernández 2010). Por la facilidad como se distribuyen en ambientes acuáticos, los agentes contaminantes pueden generar alta toxicidad en los diferentes componentes de las cadenas tróficas, en tanto que la contaminación por actividades petroleras en ambientes terrestres también puede producir un profundo deterioro; no obstante, puede aminorarse en cierta forma, ya que los componentes (orgánicos e inorgánicos) de los suelos contribuyen con su metabolismo interno a desintoxicar los ambientes afectados (HernándezValencia y Mager 2003, López-Hernández 2010), a la vez que sirven como sumideros de sustancias extrañas al ambiente.

Las características intrínsecas de los ambientes terrestres que determinan la detención o difusión de la contaminación son el tamaño de las partículas (o tamaño granulométrico) y su reactividad, representada por las característica de cargas y la capacidad adsorptiva de iones y moléculas, propiedades íntimamente relacionadas con el contenido de materia orgánica y abundancia de minerales de arcilla (Weil y Brady 2017, López-Hernández y Bates 2018). Asimismo, la actividad biológica del suelo (microorganismos y fauna del suelo) juegan un papel muy importante en los procesos de descontaminación por biorremediación (Macaulay y Rees 2014, Nagkirti et al. 2017).

En la implementación de las actividades de perforación y extracción de crudos es necesaria la utilización de un conjunto de aditivos químicos, entre los que destacan los siguientes: barita (sulfato de bario), bentonita, cal (hidróxido de calcio), gasoil, soda cáustica (hidróxido de sodio) y diferentes polímeros, lignosulfonatos, lignitos y lignina. Estas sustancias pasan a formar parte de los fluidos de perforación, por lo que se pueden convertir en potenciales agentes contaminantes para los ambientes terrestres. Para el manejo de esa situación, el Instituto de Tecnología Venezolana para el Petróleo (Intevep), en conjunto con las filiales de petróleos de Venezuela, inició un estudio sobre tratamientos y disposición de lodos provenientes de los fluidos de perforación (Liendo et al. 1991).

Para tal fin, los fluidos (aguas residuales, hidrocarburos acompañantes y desechos de la actividad de perforación) se depositan primero en una fosa, previamente impermeabilizada con una membrana epóxica para evitar la percolación del gasoil y otros contaminantes al subsuelo (Liendo et al. 1991, Arellano 2008). Posteriormente, mediante el uso de sifones se separa la fase de hidrocarburos de las aguas 
residuales (AR), siendo estas últimas transportadas a una nueva fosa denominada fosa de floculación. Ahí las AR son tratadas con un agente floculante (sulfato de aluminio); si el $\mathrm{pH}$ del agua tratada es menor a 6.5, se le añade cal con el objeto de controlar la acidez. Realizadas estas operaciones, las aguas resultantes deben ser claras y sin restos de hidrocarburos; por tanto, con condición adecuada para ser regadas al ambiente.

El tratamiento que se realiza en la fosa de floculación-coagulación tiene la finalidad de precipitar las arcillas que se encuentran como coloides cargados. El sulfato de aluminio es un buen agente floculante; sin embargo, este compuesto se hidroliza con facilidad generando bajos $\mathrm{pH}$, de ahí la necesidad del encalado (Weil y Brady 2017). No es recomendable que las aguas con $\mathrm{pH}$ ácidos se rieguen al ambiente, no sólo por su acidez per se, sino porque a bajos $\mathrm{pH}$ los metales pesados que acompañan a los lodos de perforación se encuentran en formas altamente solubles (Lindsay 2001) y constituyen un riesgo potencial de contaminación. Ha habido inquietud de parte de la industria petrolera venezolana por conocer los efectos de estas aguas residuales sobre los ecosistemas terrestres circunvecinos, especialmente en los acuíferos de zonas cercanas a las fosas de tratamientos y en los compartimentos de suelos y vegetación.

Esta contribución tiene como objetivo analizar los niveles de sales solubles y metales pesados en los suelos adyacentes a pozos petroleros, antes y después del riego con aguas floculadas y sin tratamiento de floculación.

\section{MATERIALES Y MÉTODOS}

\section{Descripción general del área de estudio}

El estudio se realizó en los alrededores de El Furrial, Estado Monagas ( $9^{\circ} 41^{\prime} \mathrm{N}$ y $63^{\circ} 27^{\prime} \mathrm{O}$, altitud $120 \mathrm{msnm}$ ), en un área de sabana afectada por la perforación y el manejo de pozos petroleros (Fig. 1). El clima de los llanos de Monagas es del tipo Awg'i según Koeppen, correspondiente a clima tropical seco con temperaturas y precipitación media anual de $26.42^{\circ} \mathrm{C}$ y $1480 \mathrm{~mm}$, respectivamente.

Los depósitos aluviales que cubren gran parte de las llanuras de Monagas se originaron en las antiguas sedimentaciones de pie de monte con largo tiempo de evolución pedogenética dando origen a Oxisoles y Ultisoles de texturas arenosas, con $\mathrm{pH}$ ácido y baja fertilidad natural (Colina et al. 1983). Los suelos de la sabana estudiada se ubican dentro del grupo OxicPaleustults, franco arenoso dentro del orden de los

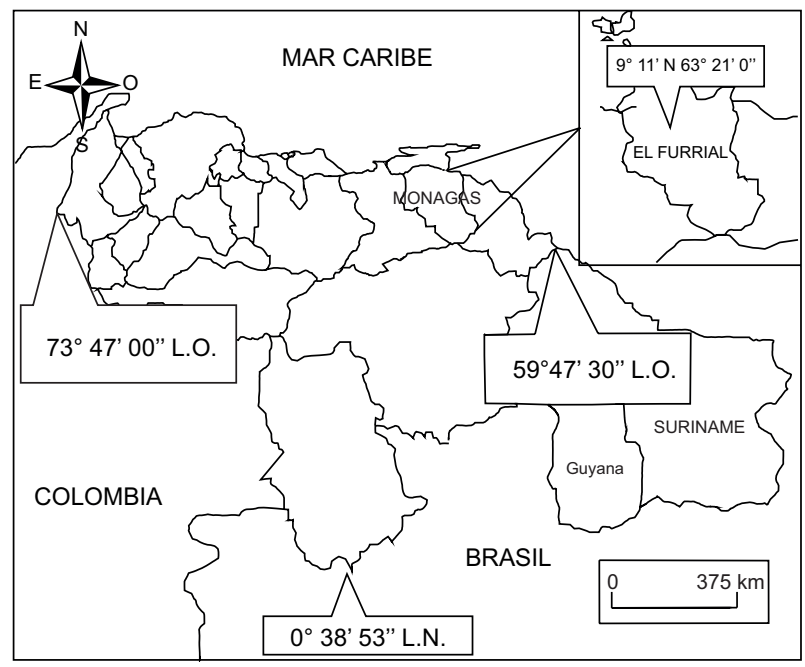

Fig. 1. Localización del área de estudio (El Furrial, Estado Monagas, Venezuela). El recuadro que indica la ubicación del Estado Monagas no está en escala.

Ultisoles. El paisaje dominante es de altiplanicie, dentro del subpaisaje de mesa conservada (mesa plano-ondulada con escurrimiento difuso) y con erosión laminar (Colina et al. 1983), mientras que la vegetación natural está constituida principalmente por gramíneas de sabanas dominadas por Trachypogon sp. y Axonopus sp.

\section{Diseño experimental}

Para los efectos de esta investigación se realizaron dos tipos de experimentos, los cuales se detallan a continuación.

\section{Experimento con riego no controlado}

Se trató de un experimento ex post facto con porciones de riego realizado por el personal de la empresa petrolera, en el cual se estudió el efecto de las aguas residuales de diferentes pozos petroleros sobre los suelos de las sabanas adyacentes. Para el experimento se seleccionaron cuatro sitios, los cuales habían sido regados durante 24 meses (Pozo Ful 2), seis meses (Pozo Ful 6), un mes (Pozo Ful 8) y cuatro días (Pozo Lagoven 5) antes del muestreo de suelos. Cabe destacar que el riego con aguas residuales se realizó como práctica habitual de desalojo de la fosa de floculación a la sabana, por lo que no se guardó registro del tiempo de riego. Como testigo se tomaron los suelos de las zonas adyacentes a los pozos.

\section{Experimento con riego controlado}

Se trató de un experimento con irrigación controlada en el cual se estudió el efecto de diferentes dosis (tiempos de riego) de aguas residuales del pozo 
petrolero Ful-15 sobre los suelos de las sabanas adyacentes. Para este último experimento fue necesario cercar un área de 0.2 ha a $400 \mathrm{~m}$ del pozo petrolero Ful-15, delimitándose en ella tres subparcelas de $200 \mathrm{~m}^{2}$ cada una. A fin de regar con las aguas de floculación las subparcelas experimentales, se colocaron dos sistemas de tuberías: la primera provenía de la fosa que contenía agua no floculada (ANF) y la segunda trasladaba agua floculada (AF). En el área experimental se procedió a la instalación de los aspersores para regar en tiempos controlados las diferentes subparcelas.

Los tratamientos de riego utilizados fueron los siguientes: dosis baja (DB), dosis mediana (DM) y dosis alta (DA), correspondientes a tiempos de riego con agua floculada de 2,4 y $8 \mathrm{~h}$, respectivamente. Igualmente, se introdujo como tratamiento adicional una subparcela regada por $2 \mathrm{~h}$ con agua de la fosa no floculada (agua cruda).

\section{Muestreo de suelos}

En ambos experimentos para la toma de muestras de suelo se abrieron calicatas tanto en los suelos regados como en una zona adyacente (no regada) correspondiente al testigo, las mismas fueron muestreadas cada $20 \mathrm{~cm}$ hasta $100 \mathrm{~cm}$ de profundidad de acuerdo con Gilabert et al. (1990). Los suelos del área con riego no controlado se muestrearon a los: 24 meses (Pozo Ful 2), seis meses (Pozo Ful 6), un mes (Pozo Ful 8) y cuatro días (Pozo Lagoven 5) de la irrigación, mientras que los suelos del área con riego controlado se muestrearon cuatro meses después de su instalación.

\section{Análisis químicos de las muestras de suelos}

Se agitó una suspensión suelo-agua (relación 1:5) durante $30 \mathrm{~min}$. Después se midió el $\mathrm{pH}$ de la solución con electrodo de vidrio. Las determinaciones de materia orgánica (MO), capacidad de intercambio (CIC) y granulometría se efectuaron por los métodos reseñados por Anderson e Ingram (1993). A fin de comparar los niveles de las formas solubles de los cationes alcalinos, alcalinotérreos, aluminio y metales pesados, antes y después del tratamiento de los suelos con las aguas de irrigación, se utilizó la solución extractora Carolina del Norte o reactivo

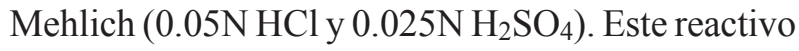
universal es particularmente apropiado para extraer formas solubles de cationes y metales pesados $(\mathrm{Na}$, $\mathrm{K}, \mathrm{Ca}, \mathrm{Mg}, \mathrm{Al}, \mathrm{Fe}, \mathrm{Mn}, \mathrm{Cu}, \mathrm{y} \mathrm{Zn}$ ) de suelos ácidos con bajo contenido de materia orgánica (Mylavarapu 2010). Todas las determinaciones se realizaron por triplicado. Los análisis de los elementos en los ex- tractos de suelos se realizaron por absorción atómica de llama en un aparato Varian Techtron AA6 (van Loon 1980). Los límites de detección de los metales pesados $\mathrm{Cu}, \mathrm{Fe}, \mathrm{Zn}, \mathrm{y} \mathrm{Mn}$ fueron 0.003, 0.005, 0.002 y $0.003 \mu \mathrm{g} / \mathrm{L}$, respectivamente.

\section{Análisis químicos de las aguas floculadas y no floculadas}

Los análisis de los elementos metálicos en las AN y las AR después de ser sometidas al proceso de floculación (AF) fueron realizados por absorción atómica en un aparato Varian Techtron AA6.

\section{Estadísticas}

Las medias de los tratamientos y sus respectivos testigos fueron comparadas por medio de pruebas $t$ con el uso del programa de computadora Statistica v. 3.1. Los datos del experimento con riego controlado fueron analizados por un análisis de varianza de dos vías con una prueba de significancia HSD-Tukey post hoc usando un paquete estadístico SPSS 13.0 con un nivel de significancia $\mathrm{p}<0.05$.

\section{RESULTADOS}

\section{Caracterización de los suelos}

Los suelos de las sabanas de El Furrial son en general de textura franco arenosa en la superficie con tendencia a incremento en el contenido de arcilla a mayor profundidad. En el Cuadro I se presentan las principales características físicas y químicas de suelos representativos de la zona correspondientes a dos de los pozos estudiados. Los $\mathrm{pH}$ de ambos suelos son bajos con un ligero incremento en el horizonte subsuperficial $(40-60 \mathrm{~cm})$. Igualmente, los contenidos de materia orgánica (0.51-0.99\%), la capacidad de intercambio catiónico $(1.2-2.0 \mathrm{cmol} /$ $\mathrm{kg}$ ) y las concentraciones de bases cambiables en todos los horizontes son muy bajas, destacando en particular la pobreza en $\mathrm{K}$, Ca y $\mathrm{Mg}$ intercambiables y los mayores niveles de Al extraíble, lo que indica una fertilidad natural muy baja. Los niveles de los micronutrientes catiónicos analizados (Fe, Mn, Zn y $\mathrm{Cu}$ ) son adecuados y están en concordancia con la acidez del perfil del suelo.

\section{Caracterización de las aguas floculadas y no floculadas}

Se realizó una caracterización preliminar de las aguas de riego usadas en la experimentación, la cual indicó que las ANF presentan altos contenidos de $\mathrm{Ba}$, $\mathrm{Na}, \mathrm{Ca}$ y $\mathrm{Al}$, cuyos niveles disminuyen drásticamente 
CUADRO I. CARACTERÍSTICAS FÍSICAS Y QUÍMICAS DE DOS SUELOS ESCOGIDOS EN POZOS PETROLEROS UBICADOS EN LOS ALREDEDORES DE EL FURRIAL, ESTADO MONAGAS, VENEZUELA.

\begin{tabular}{|c|c|c|c|c|c|c|}
\hline \multirow{2}{*}{$\frac{\text { Pozo }}{\text { Propiedades }}$} & \multicolumn{3}{|c|}{ Ful 2} & \multicolumn{3}{|c|}{ Ful 8} \\
\hline & $0-20 \mathrm{~cm}$ & $40-60 \mathrm{~cm}$ & $80-100 \mathrm{~cm}$ & $0-20 \mathrm{~cm}$ & $40-60 \mathrm{~cm}$ & $80-100 \mathrm{~cm}$ \\
\hline pH (agua) & 4.11 & 4.57 & 4.15 & 4.42 & 5.12 & 4.38 \\
\hline $\mathrm{pH}(\mathrm{KCl})$ & 4.38 & 4.37 & 4.29 & 4.30 & 4.50 & 4.31 \\
\hline$\% \mathrm{MO}$ & 0.92 & 0.51 & 0.65 & 0.99 & 0.98 & 0.77 \\
\hline CIC* & 2.0 & 1.4 & 1.9 & 1.8 & 1.2 & 1.7 \\
\hline $\mathrm{K}^{*}$ & 0.06 & 0.03 & 0.01 & 0.08 & 0.03 & 0.03 \\
\hline $\mathrm{Ca}^{*}$ & 0.51 & 0.13 & 0.25 & 0.33 & 0.15 & 0.16 \\
\hline $\mathrm{Mg}^{*}$ & 0.1 & 0.06 & 0.25 & 0.08 & 0.09 & 0.17 \\
\hline $\mathrm{Na}^{*}$ & 0.26 & 0.02 & 0.04 & 0.23 & 0.01 & 0.02 \\
\hline $\mathrm{Al}^{*}$ & 1.73 & 1.5 & 1.27 & 1.58 & 0.79 & 1.06 \\
\hline $\mathrm{Fe}^{* *}$ & 41.2 & 26.9 & 11.8 & 60.5 & 45.6 & 12.2 \\
\hline $\mathrm{Mn}^{* *}$ & 0.51 & 0.20 & 0.03 & 0.45 & 0.20 & 0.12 \\
\hline $\mathrm{Cu}^{* *}$ & 0.53 & 0.51 & 0.55 & 0.53 & 0.53 & 0.52 \\
\hline $\mathrm{Zn} * *$ & 0.49 & 0.0 & 0.1 & 0.16 & 0.06 & 0.13 \\
\hline$\%$ arena & 80.4 & 76.4 & 78.4 & 80.4 & 76.4 & 66.6 \\
\hline$\%$ limo & 6.0 & 8.0 & 2.0 & 10 & 10 & 12.0 \\
\hline$\%$ arcilla & 13.6 & 15.6 & 19.6 & 9.6 & 13.6 & 21.6 \\
\hline Textura & $\mathrm{aF}$ & $\mathrm{aF}$ & $\mathrm{aF}$ & $\mathrm{aF}$ & $\mathrm{Fa}$ & $\mathrm{Fa}$ \\
\hline
\end{tabular}

*Los valores de CIC y de $\mathrm{K}, \mathrm{Ca}, \mathrm{Mg}, \mathrm{Na}$ y Al, extraídos con el reactivo Mehlich, se expresan en cmol/kg; **los valores de $\mathrm{Fe}, \mathrm{Mn}, \mathrm{Cu} \mathrm{Y} \mathrm{Zn}$ se expresan en $\mathrm{mg} / \mathrm{kg}$.

CIC: capacidad de intercambio, MO: materia orgánica, aF: arenoso franco, Fa: franco arenoso.

con el tratamiento de coagulación-floculación. Particularmente, los altos niveles de $\mathrm{Ba}(213 \mathrm{mg} / \mathrm{L})$ y Al $(5 \mathrm{mg} / \mathrm{L})$ se reducen a trazas después del tratamiento de floculación, mientras que los contenidos de $\mathrm{Na}$, $\mathrm{Ca}, \mathrm{Fe}$ y K descienden de 183, 64, 6.2 y $3.2 \mathrm{mg} / \mathrm{L}$, a $88,49,0.5$ y $1.2 \mathrm{mg} / \mathrm{L}$, respectivamente.

\section{Efecto de los tratamientos sobre las propiedades químicas de los suelos Experimento con riego no controlado}

Para efectos de la comparación del suelo tratado con efluentes y el suelo no tratado (testigo) en el Cuadro II se incluyeron los resultados correspondientes al perfil completo $(0-100 \mathrm{~cm})$ de todos los suelos $(4$ pozos) analizados. Hay una tendencia a incrementar el $\mathrm{pH}$ en los suelos tratados con las aguas residuales, sin embargo, los valores solo fueron significativos en los horizontes más profundos $(60-100 \mathrm{~cm}$, Cuadro II). Resaltan los altos niveles de sodio que se encontraron en los suelos tratados respecto al testigo, excepto el horizonte superficial, en donde no se logró significación estadística; lo contrario se encontró en el caso del potasio en donde los valores de K extraíbles siempre fueron mayores en el suelo testigo con relación al tratamiento, aunque solo hubo significancia estadística de 0-60 cm, esta información sugiere un posible desplazamiento competitivo de este catión del complejo de cambio por el exceso de sodio en las aguas tratamientos. Los contenidos de calcio y magnesio no fueron mayormente afectados por la adición de aguas residuales a las sabanas. En lo que concierne al contenido de aluminio y los micronutrientes extraíbles con la solución Mehlich, en el caso del Al, no hubo mayores diferencias entre tratamiento y testigo de 0-60 $\mathrm{cm}$ en los perfiles analizados (Cuadro III) pero si se observaron menores valores de Al en los suelos tratados con $\mathrm{AF}$ a los 60-100 cm, información que coincide con el incremento de $\mathrm{pH}$ en esa profundidad. Para el hierro y manganeso, en general, los suelos tratados tienen menores niveles de las formas extraíbles con relación al testigo, mientras que en el caso del cobre y el zinc no se encontraron diferencias significativas entre los promedios de los testigos y los tratamientos.

\section{Experimento con riego controlado}

La acidez natural del suelo de la parcela experimental fue ligeramente neutralizada por las diferentes dosis de los efluentes (Cuadro III). A las dosis más altas las diferencias fueron más notables en los horizontes más superficiales $(0-40 \mathrm{~cm})$, mientras que a las dosis intermedia y baja el suelo tratado con efluentes mostró siempre un $\mathrm{pH}$ ligeramente 
CUADRO II. EXPERIMENTO CON RIEGO NO CONTROLADO: COMPARACIÓN DE LOS VALORES DE $\mathrm{pH}$ Y DE LOS ELEMENTOS EXTRAÍDOS (mg/kg) DE SUELOS TRATADOS CON AGUAS DE LA FOSA DE FLOCULACIÓN CON SUS RESPECTIVOS TESTIGOS EN LOS CUATRO POZOS ANALIZADOS.

\begin{tabular}{|c|c|c|c|c|c|c|}
\hline Profundidad & Parámetro & $\mathrm{pH}$ & $\mathrm{K}$ & $\mathrm{Ca}$ & $\mathrm{Mg}$ & $\mathrm{Na}$ \\
\hline \multirow[t]{2}{*}{$0-20 \mathrm{~cm}$} & $\mathrm{TE}$ & 4.93 & 10.6 & 112.4 & 18.0 & 44.4 \\
\hline & $\mathrm{C}$ & 4.88 & $29.5 *$ & 112.6 & 20.6 & 56.3 \\
\hline \multirow[t]{2}{*}{$20-40 \mathrm{~cm}$} & $\mathrm{TE}$ & 4.83 & 8.2 & 51.3 & 9.6 & $42.9 *$ \\
\hline & $\mathrm{C}$ & 4.86 & $11.1 *$ & 56.0 & 9.5 & 8.6 \\
\hline \multirow[t]{2}{*}{$40-60 \mathrm{~cm}$} & $\mathrm{TE}$ & 4.92 & 6.8 & 53.3 & 16.0 & $44.2 *$ \\
\hline & $\mathrm{C}$ & 4.88 & $11.7^{*}$ & 62.8 & 13.7 & 6.2 \\
\hline \multirow[t]{2}{*}{$60-80 \mathrm{~cm}$} & $\mathrm{TE}$ & $5.09 *$ & 9.2 & 31.7 & 18.7 & $40.0^{*}$ \\
\hline & $\mathrm{C}$ & 4.84 & 11.7 & 64.4 & 30.7 & 8.0 \\
\hline \multirow[t]{2}{*}{$80-100 \mathrm{~cm}$} & $\mathrm{TE}$ & $5.14 *$ & 7.4 & 60.1 & 26.1 & $55.3^{*}$ \\
\hline & $\mathrm{C}$ & 4.63 & 9.1 & 52.4 & 31.0 & 8.1 \\
\hline Profundidad & Parámetro & $\mathrm{Al}$ & $\mathrm{Fe}$ & $\mathrm{Mn}$ & $\mathrm{Zn}$ & $\mathrm{Cu}$ \\
\hline \multirow[t]{2}{*}{$0-20 \mathrm{~cm}$} & $\mathrm{TE}$ & 155.9 & 28.5 & 0.6 & 0.94 & 0.53 \\
\hline & $\mathrm{C}$ & 146.8 & $45.2 *$ & $9.2^{*}$ & 0.32 & 0.74 \\
\hline \multirow[t]{2}{*}{$20-40 \mathrm{~cm}$} & $\mathrm{TE}$ & 166.9 & 12.8 & 0.33 & 0.05 & 0.51 \\
\hline & $\mathrm{C}$ & 138.5 & $33.0 *$ & 3.4 & 0.12 & 0.40 \\
\hline \multirow[t]{2}{*}{$40-60 \mathrm{~cm}$} & TE & 112.6 & 10.4 & 0.1 & 0.01 & 0.46 \\
\hline & $\mathrm{C}$ & 118.7 & $28.6^{*}$ & $7.8^{*}$ & 0.04 & 0.63 \\
\hline \multirow[t]{2}{*}{$60-80 \mathrm{~cm}$} & $\mathrm{TE}$ & 71.1 & 6.3 & 0.02 & 0.04 & 0.37 \\
\hline & $\mathrm{C}$ & $124.5^{*}$ & $17.5^{*}$ & $2.64^{*}$ & 0.07 & 0.59 \\
\hline \multirow[t]{2}{*}{$80-100 \mathrm{~cm}$} & $\mathrm{TE}$ & 54.2 & 12.6 & 0.04 & 0.01 & 0.35 \\
\hline & $\mathrm{C}$ & $110.2 *$ & 11.1 & $0.3^{*}$ & 0.08 & 0.57 \\
\hline
\end{tabular}

T: fosa de floculación, TE: testigos (incluye los cuatro pozos analizados).

*Denota diferencias significativas $(\mathrm{p}=0.05)$ entre T y C.

superior al testigo. Los contenidos de $\mathrm{K}$ mostraron comportamientos muy contrastantes de acuerdo con las dosis de los tratamientos, es decir, a altas concentraciones en el suelo superficial, el contenido de K del testigo superó al tratamiento. Para el resto de los horizontes no hay diferencias entre testigo y suelo tratado. A dosis intermedia de irrigación, los contenidos de $\mathrm{K}$ del suelo no fueron afectados por las aguas residuales; sin embargo, a dosis bajas, en todos los casos, el contenido de K del suelo tratado superó al testigo. Por otro lado, en el caso del Ca y el Mg no se evidencian diferencias entre ambos tratamientos. Con relación al $\mathrm{Na}$, los efluentes incrementaron su contenido en los suelos tratados respecto del testigo en prácticamente todos los casos. Los mayores niveles de $\mathrm{Na}$ se encontraron en la dosis más alta de efluentes (Cuadro III).

El contenido de Al extraíble tiende a disminuir en las dosis alta y media de tratamiento (Cuadro IV); a dosis baja los niveles de Al fueron mayores, con una disminución del pH (Cuadro III).

Para el Fe y el Mn hay una ligera tendencia a disminuir en el tratamiento respecto del testigo, mientras que los niveles de $\mathrm{Zn}$ no fueron mayormente afectados por los diferentes tratamientos (Cuadro IV).

\section{Riego con agua no floculada}

Los suelos de las subparcelas regadas con ANF presentaron, en general, un comportamiento similar a las subparcelas regadas con agua floculada. El pH del suelo se incrementó con la adición del agua de riego, ya que la ANF presentó una carga adicional de sales producto de los insumos usados en la perforación. En asociación con el aumento de $\mathrm{pH}$, los niveles de $\mathrm{Na}$ en los suelos de las subparcelas resultaron más altos que en el testigo (Cuadro V). No se encontraron mayores cambios para $\mathrm{K}, \mathrm{Ca}$ y $\mathrm{Mg}$, mientras que en la subparcela regada con ANF hubo un incremento del 
CUADRO III. EXPERIMENTO CON RIEGO CONTROLADO: VALORES DE $\mathrm{pH}$ Y DE LOS CATIONES ALCALINOS Y ALCALINO TÉRREOS EXTRAÍDOS (mg/kg) DE SUELOS TRATADOS CON AGUAS DE LA FOSA DE FLOCULACIÓN.

\begin{tabular}{|c|c|c|c|c|c|c|}
\hline Profundidad & Elemento & $\mathrm{pH}$ & $\mathrm{K}$ & $\mathrm{Ca}$ & $\mathrm{Mg}$ & $\mathrm{Na}$ \\
\hline \multirow[t]{2}{*}{$0-20 \mathrm{~cm}$} & AT & 5.85 & 12 & 246 & 17 & 14 \\
\hline & ATE & 5.10 & 18 & 284 & 17 & 2 \\
\hline \multirow[t]{2}{*}{$20-40 \mathrm{~cm}$} & AT & 5.77 & 13 & 206 & 13 & 4 \\
\hline & ATE & 5.02 & 13 & 252 & 14 & 2 \\
\hline \multirow[t]{2}{*}{$40-60 \mathrm{~cm}$} & AT & 5.18 & 9 & 262 & 11 & 10 \\
\hline & ATE & 5.18 & 12 & 266 & 14 & 2 \\
\hline \multirow[t]{2}{*}{$60-80 \mathrm{~cm}$} & AT & 5.17 & 11 & 224 & 13 & 15 \\
\hline & ATE & 5.15 & 11 & 230 & 11 & 0.5 \\
\hline \multirow[t]{2}{*}{$80-100 \mathrm{~cm}$} & AT & 4.97 & 11 & 234 & 16 & 12 \\
\hline & ATE & 5.03 & 12 & 224 & 12 & 2 \\
\hline \multirow[t]{2}{*}{$0-20 \mathrm{~cm}$} & MT & 4.98 & 14 & 250 & 6 & 11 \\
\hline & MTE & 4.49 & 13 & 288 & 15 & 3 \\
\hline \multirow[t]{2}{*}{$20-40 \mathrm{~cm}$} & MT & 5.43 & 12 & 228 & 13 & 2 \\
\hline & MTE & 4.43 & 10 & 238 & 12 & 8 \\
\hline \multirow[t]{2}{*}{$40-60 \mathrm{~cm}$} & MT & 5.29 & 12 & 174 & 11 & 5 \\
\hline & MTE & 4.59 & 11 & 202 & 11 & 3 \\
\hline \multirow[t]{2}{*}{$60-80 \mathrm{~cm}$} & MT & 5.16 & 10 & 187 & 11 & 3 \\
\hline & MTE & 4.44 & 13 & 200 & 12 & 1 \\
\hline \multirow{2}{*}{$80-100 \mathrm{~cm}$} & MT & 5.00 & 10 & 198 & 11 & 6 \\
\hline & MTE & 4.32 & 11 & 216 & 10 & 0 \\
\hline \multirow[t]{2}{*}{$0-20 \mathrm{~cm}$} & BT & 5.60 & 15 & 232 & 14 & 2 \\
\hline & BTE & 5.34 & 11 & 310 & - & 3 \\
\hline \multirow[t]{2}{*}{$20-40 \mathrm{~cm}$} & $\mathrm{BT}$ & 5.68 & 19 & 180 & 12 & 1 \\
\hline & BTE & 5.16 & 10 & 226 & 16 & 1 \\
\hline \multirow[t]{2}{*}{$40-60 \mathrm{~cm}$} & BT & 5.39 & 16 & 175 & 11 & 8 \\
\hline & BTE & 5.08 & 14 & 244 & 13 & 0.3 \\
\hline \multirow[t]{2}{*}{$60-80 \mathrm{~cm}$} & BT & 5.30 & 20 & 186 & 11 & 6 \\
\hline & BTE & 4.93 & 9 & 228 & 12 & 1 \\
\hline \multirow[t]{2}{*}{$80-100 \mathrm{~cm}$} & BT & 5.10 & 16 & 188 & 11 & 9 \\
\hline & BTE & 4.69 & 14 & 204 & 12 & 1 \\
\hline $\mathrm{T}$ & Promedio (DE) & $\begin{array}{c}5.32 \\
(0.29)\end{array}$ & $\begin{array}{l}13.33 \\
(3.29)\end{array}$ & $\begin{array}{l}211.33 \\
(29.67)\end{array}$ & $\begin{array}{l}12.07 \\
(2.55)\end{array}$ & $\begin{array}{c}7.20 * \\
(4.51)\end{array}$ \\
\hline TE & Promedio (DE) & $\begin{array}{c}4.86 \\
(0.33)\end{array}$ & $\begin{array}{l}12.13 \\
(2.20)\end{array}$ & $\begin{array}{l}240.80 \\
(33.36)\end{array}$ & $\begin{array}{l}12.07 \\
(2.02)\end{array}$ & $\begin{array}{c}2.00 \\
(1.93)\end{array}$ \\
\hline
\end{tabular}

T: tratamiento, TE: testigo, A: alto, M: medio, B: bajo, DE: desviación estándar.

*Denota diferencias significativas $(p=0.05)$ entre T y TE. 
CUADRO IV. EXPERIMENTO CON RIEGO CONTROLADO: VALORES DEALUMINIO Y DE LOS METALES PESADOS EXTRAÍDOS $(\mathrm{mg} / \mathrm{kg})$ DE SUELOS TRATADOS CON AGUAS DE LA FOSA DE FLOCULACIÓN.

\begin{tabular}{|c|c|c|c|c|c|}
\hline Profundidad & Elemento & $\mathrm{Al}$ & $\mathrm{Fe}$ & $\mathrm{Mn}$ & $\mathrm{Zn}$ \\
\hline \multirow[t]{2}{*}{$0-20 \mathrm{~cm}$} & AT & 55 & 36 & 2 & 0.7 \\
\hline & ATE & 79 & 32 & 0.8 & 0.7 \\
\hline \multirow[t]{2}{*}{$20-40 \mathrm{~cm}$} & $\mathrm{AT}$ & 117 & 20 & 0.3 & 0.6 \\
\hline & ATE & 241 & 20 & 0.3 & 0.4 \\
\hline \multirow{2}{*}{$40-60 \mathrm{~cm}$} & AT & 152 & 8 & 0.3 & 2.0 \\
\hline & ATE & 490 & 12 & 0.0 & 0.4 \\
\hline \multirow[t]{2}{*}{$60-80 \mathrm{~cm}$} & AT & 119 & 7 & 0.0 & 0.8 \\
\hline & ATE & 190 & 12 & 0.0 & 0.3 \\
\hline \multirow[t]{2}{*}{$80-100 \mathrm{~cm}$} & AT & 69 & 9 & 0.0 & 0.5 \\
\hline & ATE & - & 9 & 0.0 & 0.8 \\
\hline \multirow[t]{2}{*}{$0-20 \mathrm{~cm}$} & MT & 76 & 36 & 1.0 & 0.6 \\
\hline & MTE & 84 & 45 & 1.6 & 0.8 \\
\hline \multirow[t]{2}{*}{$20-40 \mathrm{~cm}$} & MT & 153 & 29 & 0.5 & 0.6 \\
\hline & MTE & 137 & 38 & 0.5 & 0.6 \\
\hline \multirow[t]{2}{*}{$40-60 \mathrm{~cm}$} & MT & 263 & 12 & 0 & 0.4 \\
\hline & MTE & 300 & 16 & 0 & 3.0 \\
\hline \multirow[t]{2}{*}{$60-80 \mathrm{~cm}$} & MT & 134 & 11 & 0 & 0.5 \\
\hline & MTE & 199 & 9 & 0 & 0.8 \\
\hline \multirow[t]{2}{*}{$80-100 \mathrm{~cm}$} & MT & 22 & 7 & 0 & 0.5 \\
\hline & MTE & 37 & 10 & 0 & 0.5 \\
\hline \multirow[t]{2}{*}{$0-20 \mathrm{~cm}$} & BT & 92 & 31 & 1 & 3 \\
\hline & BTE & 67 & 41 & 2.5 & 0.9 \\
\hline \multirow[t]{2}{*}{$20-40 \mathrm{~cm}$} & BT & 224 & 32 & 0.4 & 1 \\
\hline & BTE & 133 & 29 & 0 & 0.6 \\
\hline \multirow[t]{2}{*}{$40-60 \mathrm{~cm}$} & BT & 364 & 9 & 0.3 & 0.5 \\
\hline & BTE & 139 & 12 & 0 & 0.5 \\
\hline \multirow[t]{2}{*}{$60-80 \mathrm{~cm}$} & BT & 206 & 8 & 0.3 & 1.0 \\
\hline & BTE & 132 & 11 & 0 & 0.6 \\
\hline \multirow[t]{2}{*}{$80-100 \mathrm{~cm}$} & BT & 22 & 7 & 0 & 0.8 \\
\hline & BTE & 146 & 7 & 0 & 0.7 \\
\hline $\mathrm{T}$ & $\begin{array}{l}\text { Promedio } \\
\text { (DE) }\end{array}$ & $\begin{array}{l}137.87 \\
(94.30)\end{array}$ & $\begin{array}{c}17.47 \\
(11.78)\end{array}$ & $\begin{array}{c}0.41 \\
(0.55)\end{array}$ & $\begin{array}{c}0.90 \\
(0.70)\end{array}$ \\
\hline $\mathrm{TE}$ & $\begin{array}{l}\text { Promedio } \\
\text { (DE) }\end{array}$ & $\begin{array}{c}111.8 \\
(73.22)\end{array}$ & $\begin{array}{c}20.58 \\
(13.13)\end{array}$ & $\begin{array}{c}1.15 \\
(0.74)\end{array}$ & $\begin{array}{c}0.77 \\
(0.64)\end{array}$ \\
\hline
\end{tabular}

T: tratamiento, TE: testigo, A: alto, M: medio, B: bajo, DE: desviación estándar. *Denota diferencias significativas $(p=0.05)$ entre T y TE. 
CUADRO V. EXPERIMENTO CON RIEGO NO CONTROLADO: COMPARACIÓN DE LOS VALORES DE pH Y DE LOS CATIONES ALCALINOS Y ALCALINO TÉRREOS Y ALUMINIO EXTRAÍDOS (mg/kg) DE SUELOS TRATADOS CON AGUAS DE LA FOSA CON AGUA NO FLOCULADA(ANF) CON SUS RESPECTIVOS TESTIGOS (TE).

\begin{tabular}{llllllll}
\hline Profundidad & & $\mathrm{pH}$ & $\mathrm{K}$ & $\mathrm{Ca}$ & $\mathrm{Mg}$ & $\mathrm{Na}$ & $\mathrm{Al}$ \\
\hline $0-20 \mathrm{~cm}$ & ANFT & $5.28^{*}$ & 16 & 216 & 12 & 2 & 143 \\
& AFNTE & 4.86 & 27 & 218 & 14 & 1 & 133 \\
\hline $20-40 \mathrm{~cm}$ & ANFT & $5.20^{*}$ & 13 & 180 & 11 & $6 *$ & $261^{*}$ \\
& ANFTE & 4.75 & 13 & 297 & 17 & 2 & 104 \\
\hline $40-60 \mathrm{~cm}$ & ANFT & $5.37 *$ & 9 & 184 & 11 & $6 *$ & $303^{*}$ \\
& ANFTE & 4.80 & 14 & 222 & 12 & 2 & 76 \\
\hline $60-80 \mathrm{~cm}$ & ANFT & 4.74 & 11 & 190 & 11 & $12^{*}$ & 35 \\
& ANFTE & 4.74 & 12 & 224 & 11 & 0.6 & 51 \\
\hline $80-100 \mathrm{~cm}$ & ANFT & 4.68 & 17 & 210 & 14 & $10^{*}$ & 0.4 \\
& ANFTE & 4.77 & 12 & 210 & 11 & 49 \\
\hline
\end{tabular}

ANF: aguas no floculadas, TE: testigo.

*Denota diferencias significativas $(\mathrm{p}=0.05)$ entre T y TE.

contenido de $\mathrm{Al}$ asociado con los altos niveles de este elemento en las aguas sin tratamiento. Los metales pesados analizados en estas subparcelas (valores no presentados) no mostraron diferencias al compararse tratamiento y testigo.

\section{DISCUSIÓN}

La explotación petrolera es una actividad que implica procesos complejos que tienen un gran potencial para afectar al ambiente, dañando los ecosistemas y en casos extremos hasta la salud de las poblaciones humanas (Gay et al. 2010). Relativamente pocos estudios han abordado el impacto de la disposición de fluidos de perforación petrolera en el sistema suelo-planta-agua. Algunas investigaciones han señalado que los altos niveles de sales solubles, metales pesados y residuos de petróleo presentes en los fluidos de perforación son perjudiciales para la calidad del suelo y el crecimiento de las plantas (Wojtanowicz 2008, Zvomuya et al. 2011). Sin embargo, la información no es del todo concluyente, ya que en algunos estudios se reporta un impacto positivo (o nulo) de los fluidos cuando éstos se aplican en dosis bajas a suelos de regiones áridas con texturas gruesas, como consecuencia del incremento de $\mathrm{pH}$, la potencial adición de micronutrientes y el mejoramiento general de las propiedades del suelo (Bauder et al. 2005, Magalhães et al. 2014, Yao y Naeth 2014). Son pocos los estudios que se han centrado en la liberación de elementos tóxicos de los fluidos de perforación de pozos petroleros, y su efecto en el suelo y los ecosistemas acuáticos (Zvomuya et al. 2011, Purser y Thomsen 2012).

Los suelos analizados (tanto los correspondientes a los diferentes pozos estudiados como el suelo de la parcela experimental) corresponden en general a Ultisoles franco arenosos, por lo que todos los testigos analizados presentaron bajos niveles de $\mathrm{pH}$ (4.10-5.20), producto de un lavado intenso de los cationes básicos $\left(\mathrm{Ca}^{++}, \mathrm{Mg}^{++}, \mathrm{Na}^{+}, \mathrm{K}^{+}\right)$y el predominio de los cationes ácidos $\left(\mathrm{Al}^{3+}, \mathrm{H}^{+}\right)$, tanto en los sitios de intercambio como en la solución del suelo. El contenido de sales de las aguas provenientes de efluentes de pozos petroleros que son regadas a las sabanas es mucho más alto que el que corresponde normalmente a las aguas de lluvias. La acidez natural de esos suelos fue ligeramente neutralizada con las dosis utilizadas de los efluentes (efluente tratado y efluente no tratado o crudo). Al comparar los diferentes tratamientos y los testigos, se encontraron diferencias estadísticamente significativas para el $\mathrm{pH}$. Es importante señalar que, en casi todos los casos, las muestras de suelo tratadas con las aguas de perforación fueron recolectadas varios meses después de haber realizado el riego, lo que indica que aun transcurrido ese lapso de tiempo se observa el efecto de los tratamientos sobre el $\mathrm{pH}$ de los suelos de la zona. Resultados similares fueron presentados en ensayos con lodos y aguas de perforación en suelos cultivados (Whitfill y Boyd 1987). 
Con base en los resultados obtenidos para los macroelementos y microelementos analizados, se concluye que después de un tiempo de haber efectuado el riego no hay mayor alteración de la composición química del suelo tratado con los efluentes que provienen de la fosa de coagulación-floculación, ya que los análisis estadísticos de los resultados obtenidos para el contenido de esos elementos en los suelos y sus respectivos testigos señalan que no hubo diferencias estadísticamente significativas. Asimismo los altos niveles de Ca presentes en los efluentes provenientes de los pozos petroleros no afectaron de manera significativa el nivel del Ca soluble en el suelo.

En el experimento con riego controlado, el $\mathrm{Na}$ y el $\mathrm{Al}$ fueron los únicos que presentaron diferencias estadísticamente significativas $(\mathrm{Na}, \mathrm{F}=8.05 ; \mathrm{Al}, \mathrm{F}$ $=3.11 ; \alpha=0.05$ ) cuando se compararon los suelos tratados con sus respectivos testigos. Esto se reflejó en una mayor concentración de $\mathrm{Na}$ y en algunos casos una disminución del contenido de Al luego del tratamiento con el efluente (Cuadros III y IV). Sin embargo, según reportes de la literatura los valores de Al y metales pesados en los suelos están generalmente sometidos a una alta variabilidad (VallejoTorres y López-Hernández 1993), lo que dificulta la interpretación de resultados. En el caso del Ca y el $\mathrm{Mg}$, estos mayores niveles no constituirían problema alguno para el ambiente; al contrario, debido a los bajos $\mathrm{pH}$ de estos suelos, dichos nutrientes actuarían como sustancias encalantes, mejorando la fertilidad natural del suelo y disminuyendo los niveles de $\mathrm{Al}$ soluble. No se puede afirmar lo mismo del $\mathrm{Na}$, ya que éste no es considerado esencial para el desarrollo de las plantas, según reportes de la literatura, aunque sí se considera dentro de la dieta del ganado. Se ha señalado que los fluidos que contienen altas concentraciones de sales causan efectos significativos en el suelo y la vegetación (Sampaio et al. 2015). Los niveles de $\mathrm{Na}$ que se incorporan podrían constituir un problema ambiental delicado si los mismos se acumulan en suelos con mal drenaje, lo que podría inducir una salinización sódica. Ahora bien, en el caso específico del área de estudio, aunque se encontraron niveles importantes de $\mathrm{Na}$ en los suelos tratados, que contribuyen a desplazar el K del complejo de cambio en los suelos regados con AR, se debe descartar el peligro de salinización, ya que este elemento no tiende a permanecer por mucho tiempo en el perfil de los suelos estudiados debido a su buen drenaje y a la adecuada precipitación de la región. Tampoco los niveles de Ca y el $\mathrm{pH}$ fueron lo suficientemente altos para que se generaran sales carbonatadas. Más aún, el incremento del $\mathrm{pH}$ de los suelos tratados con dosis importantes del efluente (dosis alta en el experimento con riego controlado), no llega a valores extremos, lo que corrobora que no hay riesgo de salinización.

\section{CONCLUSIONES}

El tratamiento de coagulación-floculación reduce las concentraciones de $\mathrm{Ca}, \mathrm{Na}$, $\mathrm{Ba}$ y $\mathrm{Al}$ presentes en los fluidos de perforación. En las AR tratadas se observa un efecto positivo, ya que el $\mathrm{pH}$ de los suelos ácidos aumenta; asimismo, las concentraciones de $\mathrm{Na}$ de las AR tratadas con la técnica de coagulaciónfloculación disminuyen los niveles de $\mathrm{Al}$ soluble en el suelo. Tampoco se encontraron niveles altos de metales pesados (Fe, Mn, Cu y Zn) en los suelos, ya que las formas solubles de esos elementos decaen con la concomitante subida de $\mathrm{pH}$.

No existe un riesgo ambiental potencial debido a los niveles de $\mathrm{Na}$ soluble que las $\mathrm{AR}$ transfieren al ambiente, ya que el buen drenaje interno de estos Ultisoles y la adecuada precipitación de la zona permiten el pronto desalojo de sales sin peligro de inducción de salinización; sin embargo, se recomienda una estricta vigilancia, en particular de los niveles de $\mathrm{pH}$ de las aguas residuales antes de ser regadas a las sabanas circunvecinas.

\section{AGRADECIMIENTOS}

Se agradece la colaboración financiera del Instituto de Tecnología Venezolana para el Petróleo (Intevep). Los autores desean expresar su gratitud a dos revisores anónimos que contribuyeron a mejorar la edición final del manuscrito con sus apropiados comentarios.

\section{REFERENCIAS}

Anderson J.M. e Ingram J.S. (1993). Tropical soil biology and fertility: A handbook of methods. 2a ed. Wallingford, Reino Unido, $221 \mathrm{pp}$.

Arellano T. (2008). Manejo integral de fosas de hidrocarburos generadas por la actividad petrolera venezolana. Tesis de Maestría. Universidad de la Fuerza Armada Nacional, Caracas, Venezuela, 119 pp.

Bauder T.A., Barbarick K.A., Ippolito J.A., Shanahan F.J. y Ayers P.D. (2005). Soil properties affecting wheat yields following drilling fluids application. J. Environ. Qual. 34, 1687-1696. https://doi.org/10.2134/ jeq2004.0384 
Colina P., Rivera J. y Vásquez G. (1983). Estudio preliminar de suelos. Llanos Altos del Estado Monagas. Informe. Ministerio del Ambiente y Recursos Naturales Renovables, Caracas, Venezuela, 105 pp.

Gay J., Shepherd O., Thyden M. y Whitman M. (2010). The health effects of oil contamination: A compilation of research. Worcester Polytechnic Institute, Worcester MA, EUA, $211 \mathrm{pp}$.

Gilabert B.J. de, López R.I. de y Pérez R.R. de (1990). Manual de métodos y procedimientos de referencia. Análisis de suelos para diagnóstico de fertilidad. Ministerio de Agricultura y Cria-Fondo Nacional de Investigaciones Agronómicas y Pecuarias-Centro Nacional de Investigaciones Pecuarias (Serie D. 26). Maracay, Venezuela, 164 pp.

Hernández-Valencia I. y Mager D. (2003). Uso de Panicum maximum y Brachiaria brizantha para fitorremediar suelos contaminados con un crudo de petróleo liviano. Bioagro 153, 149-156.

Konečný F., Boháček Z., Müller P., Kovářová M. y Sedláčková I. (2003). Contamination of soils and groundwater by petroleum hydrocarbons and volatile organic compounds - Case study: Elslav Brno. Bulletin of Geosciences 78, 225-239.

Liendo F., Serrano C., Urich J., Díaz A., Morales G. y López D. (1991). Tratamiento y disposición de efluentes de perforación en el área de El Furrial, Edo. Monagas, Venezuela. Revista Técnica Intevep 11, 173-182.

Lindsay W.L. (2001). Chemical equilibria in soils. John Wiley and Sons, Nueva York, EUA, 472 pp.

López-Hernández D. (2010). Impacto y resiliencia en indicadores de calidad de suelos en sabanas y morichales de los llanos orientales venezolanos contaminados por un derrame petrolero. En: Contaminación, descontaminación y restauración ambiental en Ibero América (Gallardo Lancho J.F., Coord.). Sociedad Ibero Americana de Física y Química Ambiental, Salamanca, España, pp. 165-182.

López-Hernández D. y Bates J. (2018). Determinación de áreas superficiales específicas y parámetros asociados en suelos venezolanos seleccionados. Revista de la Facultad de Agronomía de la Universidad del Zulia 35, 26-39.

Macaulay B.M. y Rees D. (2014). Bioremediation of oil spills: A review of challenges for research advancement. Annals of Environmental Science 8, 9-37.

Magalhães M.O.L., Amaral Sobrinho N.M.B., Zonta E., Simões B.F., Mattos A.G., Tolón-Becerra A. y LastraBravo X.B. (2014). The effects of oil well drilling cuttings on soil and rice (Oriza sativa) under two redox conditions. Bull. Environ. Contam. Toxicol. 92, 311-316. https://doi.org/10.007/s00128-014-1196-7

Mylavarapu R.S. (2010). Diagnostic nutrient testing. Hort. Tech. 20, 19-22. https://doi.org/10.21273/HORTTECH.20.1.19

Nagkirti P., Shaikh A., Vasudevan G., Paliwal V. y Dhakephalkar P. (2017). Bioremediation of terrestrial oil spills: Feasibility Assessment. En: Optimization and applicability of bioprocesses (Purohit H., Kalia V., A Vaidya. y Khardenavis A., Eds.). Springer, Singapur, 141-173. https://doi.org/10.1007/978-98110-6863-8_7

Purser A. y Thomsen L. (2012). Monitoring strategies for drill cutting discharge in the vicinity of cold-water coral ecosystems. Mar. Pollut. Bull. 64, 2309-2316. https://doi.org/10.1016/j.marpolbul

Sampaio Junior J., do Amaral N.M.B., Zonta E. y Magalhães M.O.L. (2015). Barium and sodium in sunflower plants cultivated in soil treated with wastes of drilling of oil well. Rev. Bras. Eng. Agríc. Amb. 19, 11001106. https://doi.org/10.1590/1807-1929/agriambi. v19n11p1100-1106

Vallejo-Torres O. y López-Hernández D. (1993). Fracciones químicas de micronutrientes en el suelo en un agroecosistema de caña de azúcar. Turrialba 43 (4), 276-283.

Van Loon J.C. (1980). Analytical atomic spectroscopy. Selected methods. Academic Press, Nueva York, EUA, 331 pp.

Weil R.R y Brady N.C. (2017). The nature and properties of soil. 15th ed. Pearson Press, Nueva York, EUA, $1104 \mathrm{pp}$.

Whitfill D.L. y Boyd P.A. (1987). Soil farming of oil mud drill cutting. Conference of the International Association of Drilling Contractors. Nueva Orleans, LA, EUA, 429-438.

Wojtanowicz A.K. (2008). Environmental control of drilling fluids and produced water. En: Environmental technology in the oil industry (Orszulik S.T., Ed.). Springer, Hampshire, Reino Unido, 77-121. https:// doi.org/10.1007/978-3-319-24334-4

Yao L. y Naeth M.A. (2014). Soil and plant response to unused potassium silicate drilling fluid application. Ecol. Eng. 73, 461-468. https://doi.org/10.1016/j. ecoleng.2014.09.110

Zvomuya F., Larney F.J., Willms W.D., Beck R.K. y Olson A.F. (2011). Vegetation response to a one-time spent drilling mud application to Semiarid Mixed-Grass Prairie. Range Ecol. Manag. 64, 375-383. https://doi. org/10.2111/REM-D-10-00028.1 